\title{
Capital Psicológico de los Trabajadores en España. Análisis Factorial Confirmatorio del PCQ-12
}

\author{
Psychological Capital of Spanish Workers: Confirmatory Factor Analysis of PCQ-12
}

\author{
Mªnaculada López-Núñez ${ }^{1}$, Saul Neves de Jesús ${ }^{2}$, João Viseu ${ }^{3}$, Soraya Santana-Cárdenas ${ }^{4}$
}

\begin{abstract}
Resumen
Antecedentes: el concepto de capital psicológico positivo, derivado del estudio del comportamiento organizacional positivo, hace referencia a un estado psicológico caracterizado por autoeficacia, esperanza, resiliencia y optimismo, que puede ser desarrollado y que se relaciona con el rendimiento laboral. Método: este estudio analiza la fiabilidad y estructura factorial del cuestionario de Capital Psicológico (PCQ-12) en una muestra de trabajadores españoles $(N=339)$ de distintos sectores profesionales a través de la técnica del análisis factorial confirmatorio (CFA). Resultados: los análisis muestran un modelo de ajuste compuesto por cuatro factores interrelacionados frente a un modelo de segundo orden propuesto por los autores del instrumento. Se discuten los resultados de ajuste factorial y fiabilidad en relación con varios estudios que difieren con el modelo encontrado, así como sus limitaciones y contribución. Conclusiones: se plantea la necesidad de contemplar la influencia y sensibilidad del instrumento a las diferencias culturales tanto geográficas como organizacionales.
\end{abstract}

Palabras clave: capital psicológico, PCQ-12, análisis factorial confirmatorio, influencia cultural

\begin{abstract}
Background: The concept of positive psychological capital draws from the study of positive organizational behavior and refers to a psychological state characterized by self-efficacy, hope, resilience, and optimism that can be developed and related to job performance. Methods: This paper analyzed the reliability and factorial structure of the Psychological Capital Questionnaire-12 (PCQ-12) in a sample composed by Spanish workers $(N=339)$, from different professional sectors, through a confirmatory factor analysis (CFA). Results: The analysis assessed a model with four-correlated factors competing with a second-order model proposed by the authors of this instrument. The results of the adjustment and reliability values of the dimensions are discussed in light of various studies which differ from our model or support it along with its limitations and contributes. Conclusion: Our results indicate the need to contemplate this instrument's sensitivity towards cultural differences, both geographical and organizational.
\end{abstract}

Keywords: Psychological capital, PCQ-12, confirmatory factor analysis, cross-cultural

\footnotetext{
${ }^{1}$ Doctora en psicología. Universidad Complutense de Madrid. Profesora Titular Interina en la Facultad de Psicología. Campus de Somosaguas, 28223. Madrid, España. Tel.: 913043231. Correo: mariai04@ucm.es

${ }^{2}$ Professor Catedrático da Universidade do Algarve. Presidente do Conselho Científico da Faculdade de Ciências Humanas e Sociais. President of the Scientific Council in the Faculty of Human and Social Sciences. Presidente do Centro de Investigação sobre Espaço e Organizações (CIEO).Universidade do Algarve, Campus de Gambelas, 8005-139 Faro, Portugal. Correo: snjesus@ualg.pt

${ }^{3}$ Professor Assitente da Universidade do Algarve. Centro de Investigação sobre Espaço e Organizações (CIEO). Campus de Gambelas, 8005-139 Faro, Portugal. Correo: jnviseu@ualg.pt

${ }^{4}$ Doctora en psicología. Centro Universitario del Sur (CUSUR). Universidad de Guadalajara. Profesora Investigadora. Av. Enrique Arreola Silva No. 883, colonia centro C.P. 49000, Ciudad Guzmán, Jalisco, México. Tel.: +52(341)5752222. Correo: soraya@cusur.udg.mx
} 


\section{Introducción}

El enfoque de la psicología positiva (Seligman \& Csikszentmihalyi, 2000) surge al considerar que el estudio de la psicología se había centrado durante mucho tiempo en lo que estaba mal en el individuo ignorando el papel protector de las fortalezas humanas (Myers, 2000).

Esta perspectiva de lo positivo no es nueva, ya en la corriente de la psicología humanista, autores como Rogers (1961) y Maslow (1954) hablaban de la necesidad de recoger una visión positiva dirigida al bienestar psicológico (Barrera-Guzmán \& Flores-Galaz, 2015).

El enfoque en la debilidad no era exclusivo de la psicología clínica, también en el ámbito organizacional existía un sesgo centrado en lo negativo, en el estrés laboral. Investigadores de este campo empezaron a plantear la oportunidad de adoptar una orientación positiva, lo que dio lugar a la aparición del estudio del comportamiento organizacional positivo (COP) (Luthans, 2002a, 2002b).

Esta visión se caracteriza por ser proactiva, centrada en las fortalezas y en la consideración de los trabajadores como la principal ventaja competitiva de una organización (Avey, Luthans, \& Youssef, 2010; Youssef-Morgan, 2014). Recientemente, Cabanas y Sánchez-González (2016, p.107) hablaban de la "inversión de la pirámide de necesidades" para situar a la felicidad como una de las necesidades que se deben alcanzar para prosperar en la vida en general y especialmente en el ámbito laboral.

Del desarrollo de la psicología positiva y del comportamiento organizacional nace un nuevo campo de conocimiento: la psicología de la salud ocupacional positiva (PSOP) (Bakker \& Rodríguez Muñoz, 2012), que estudia cómo los recursos laborales y personales pueden proteger de los riesgos y fomentar la salud en el lugar de trabajo (Bakker \& Demerouti, 2013), mejorar el rendimiento y el bienestar (Torrente, Salanova, Llorens, \& Schaufeli, 2012).

\section{Comportamiento Organizacional Positivo (COP)}

El comportamiento organizacional positivo (COP) se define como: "el estudio y aplicación de las fortalezas y capacidades psicológicas positivas que pueden ser medidas, desarrolladas y gestionadas para mejorar el rendimiento laboral" (Luthans, 2002b, p. 54). Estas capacidades psicológicas deben tener una extensa fundamentación teórica y empírica, medidas validadas, y lo más importante: capacidad de contribuir al rendimiento en el lugar de trabajo.

Deben ser flexibles y abiertas al desarrollo. Se trata por tanto, de estados psicológicos positivos a los que llamaron state like (Luthans, Avolio, Avey, \& Norman, 2007). Estos estados no son independientes de los rasgos psicológicos. Frente a la dicotomía clásica, los autores presentan un continuo a lo largo del cual los rasgos y estados se sitúan en función de su estabilidad y flexibilidad. Entre las capacidades positivas (state like) Luthans, Avolio et al., (2007) dedicaron una extensa investigación a la autoeficacia, esperanza, optimismo y resiliencia.

\section{Autoeficacia}

Basada en la teoría de Bandura (Bandura, 1997), se define como la seguridad que el individuo tiene en sus capacidades para movilizar los recursos cognitivos y los comportamientos necesarios para conseguir altos niveles de rendimiento (Stajkovic \& Luthans, 1998).

De las cuatro capacidades estudiadas, Luthans (2002a) considera que la autoeficacia es la más relevante porque tiene una gran fundamentación teórica, un buen apoyo empírico como estado psicológico, y su relación con diversos criterios de rendimiento está bien establecida (Luthans \& Ibrayeva, 2006).

\section{Esperanza}

Es un estado motivacional positivo derivado de la sensación de poder alcanzar el éxito por la planificación y acción planteadas (Snyder, Irving, \& Anderson, 1991). Su naturaleza de estado psicológico ha sido apoyada empíricamente y a través de diferentes intervenciones se ha demostrado su relación con aspectos del COP (Martínez \& Cassaretto, 2012; Peterson \& Luthans, 2003).

\section{Optimismo}

Se define como un estilo atribucional que explica los sucesos positivos como personales, permanentes y generalizados, y los negativos como externos, temporales y específicos (Peterson 
\& Steen, 2002). El optimismo ha sido asociado con un gran número de resultados positivos, rendimiento laboral y liderazgo (Schulman, 1999).

\section{Resiliencia}

La resiliencia es la capacidad para enfrentarse a las adversidades y recuperarse (Block \& Kremen, 1996). Desde el enfoque del COP, la resiliencia es una dimensión proactiva que permite que la adversidad sea vista como una oportunidad para el crecimiento. La resiliencia se ha mostrado predictor de resultados positivos en el entorno laboral y abierta al desarrollo (Sánchez-Teruel \& Robles-Bello, 2014; Waite \& Richardson, 2004).

\section{Capital Psicológico Positivo}

La revisión teórica y empírica mostró suficiente evidencia de que estas cuatro capacidades reunían los criterios para ser aspectos relevantes del COP. De la investigación sobre su interacción surge un constructo central que Luthans, Youssef-Morgan y Avolio (2015, p. 2) denominaron: capital psicológico positivo (CapPsy) y que definieron como: un estado psicológico positivo de desarrollo que se caracteriza por: (1) tener confianza (autoeficacia) para hace el esfuerzo necesario y conseguir el éxito en tareas desafiantes; (2) hacer atribuciones positivas (optimismo) para obtener el éxito en el presente y futuro; (3) perseverar en los objetivos $\mathrm{y}$, cuando sea necesario, reorientarlos (esperanza) para alcanzar el éxito; y (4) cuando aparezcan los problemas y adversidades, resistir y recuperarse (resiliencia) para lograr el éxito.

Gran número de investigaciones demuestran que el capital psicológico se relaciona positivamente con: el compromiso organizacional (Luthans \& Jensen, 2005), la satisfacción laboral (Luthans, Avolio et al., 2007), la creatividad (Sweetman, Luthans, Avey, \& Luthans, 2011), el rendimiento (Youssef-Morgan \& Luthans, 2015) y el bienestar psicológico (Baron, Franklin, \& Hmieleski, 2013).

Por otro lado, se han encontrado evidencias que lo relacionan negativamente con: el absentismo, la rotación y conductas contraproducentes en el trabajo (Avey, Wernsing, \& Luthans, 2008).

\section{La Evaluación y Medida (Measurement) del} Capital Psicológico Positivo

Para su evaluación y medida, Luthans, Youssef y Avolio (2007) desarrollaron y validaron el Cuestionario de Capital Psicológico PCQ (por su siglas en inglés), compuesto por 24 ítems (PCQ-24) y una versión reducida de 12 ítems (PCQ-12), derivado de medidas consolidadas de esperanza (Snyder et al., 1996), eficacia (Parker, 1998), resiliencia (Wagnild \& Young, 1993), y optimismo (Scheier \& Carver, 1985) adaptadas para su aplicación en el contexto laboral.

Los autores señalaron que la estructura factorial del PCQ responde a un modelo compuesto por cuatro factores de primer orden (autoeficacia, esperanza, resiliencia y optimismo) $\mathrm{y}$ un factor de segundo orden (Capital Psicológico). Esta estructura ha sido confirmada por varios estudios (e.g. Avey, Reichard, Luthans, \& Mhatre, 2011) y en distintos contextos culturales para la versión de 24 y 12 ítems (Alessandri, Borgogni, Consiglio, \& Mitidieri, 2015; Azanza, Domínguez, Moriano, \& Molero, 2014; Badran \& Youssef-Morgan, 2015; Caza, Bagozzi, Woolley, Levy, \& Caza, 2010; LeónPérez, Antino, \& León-Rubio, 2017; Rus, Baban, Jesus, \& Andrei, 2012; Wernsing, 2014). Sin embargo otras investigaciones no han encontrado los mismos resultados (Luthans, Avey, ClappSmith, \& Li, 2008; Rego, Marques, Leal, Sousa, \& Pina e Cunha, 2010; Viseu et al., 2012).

Estos datos indican la necesidad de ampliar la investigación de las propiedades psicométricas y factoriales del instrumento para clarificar la relación entre sus componentes. Además, algunos autores (e.g. Avey, 2014; Newman, Ucbasaran, Zhu, \& Hirst, 2014) indican que se deben explorar los antecedentes del PsyCap, así como el papel mediador y moderador de este constructo en el comportamiento y rendimiento laboral.

Dada la escasez de trabajos con el cuestionario PCQ-12 y la existencia de un sólo estudio con población española (León-Pérez et al., 2017), este trabajo pretende aportar nueva evidencias en cuanto a su análisis factorial y propiedades psicométricas, y ahondar en un mejor conocimiento sobre el ajuste de los factores que componen el constructo de capital psicológico evaluado con este instrumento. 
Este nuevo trabajo de validación en población española es especialmente útil para aclarar algunas lagunas de trabajos anteriores (e.g. LeónPérez et al., 2017). León-Pérez et al. (2017) utilizaron una muestra compuesta exclusivamente por trabajadores de una misma organización, lo que supone una limitación y un posible sesgo en los resultados. También Azanza et al. (2014), al referirse a los instrumentos para medir el capital psicológico positivo, sugieren que muestras de sectores específicos pueden indicar una composición factorial particular, por lo que se aconseja el uso de muestras compuestas por profesionales de varios sectores. Este mismo hecho fue señalado por Viseu et al. (2012), al indicar que son necesarios más estudios de validación sobre este instrumento debido a posibles variaciones interculturales e inter organizacionales del constructo de capital psicológico positivo. En la misma línea Wernsing (2014) señala que la cuestión fundamental en estos momentos es la relativa a la equivalencia a través de distintas culturas de las propiedades psicométricas del constructo.

Además de todo lo anterior, este trabajo tiene interés porque aporta nuevo conocimiento a una de las principales críticas que se ha hecho a la medida del capital psicológico positivo y es que, a pesar de que hay mucha investigación en torno a su relación con el COP, se hacen necesarios más estudios que evalúen su conceptualización y propiedades psicométricas. Los meta-análisis existentes (Avey, Reichard, et al., 2011) no evalúan estos aspectos y revisiones de los propios autores del constructo (Youssef \& Luthans, 2011, 2012) también parecen omitir la evaluación crítica de sus características psicométricas (Dawkins, Martin, Scott, \& Sanderson, 2013).

Así pues, parece relevante y necesario la realización de una nueva validación del PCQ-12 para la población española recurriendo a una muestra que esté compuesta por trabajadores de diferentes organizaciones y sectores, como mostramos en este trabajo.

Por otro lado, y dado que la mayoría de los estudios que avalan el capital psicológico positivo lo hacen a través del PCQ-24 (e. g. Azanza et al., 2014), se hace necesario, tal y como señala Luthans et al. (2015), validaciones de la versión reducida del PCQ para facilitar la medición del capital psicológico positivo en el contexto organizacional, donde las restricciones de tiempo son elevadas y es importante contar con instrumentos de evaluación breves

\section{Método}

\section{Participantes}

Para la selección de los participantes se contactó con organizaciones de diversos sectores de actividad y se solicitó su participación en este estudio. La participación en esta investigación fue voluntaria no habiendo criterios de exclusión en la selección de la muestra, y garantizando la total confidencialidad de los datos obtenidos.

Un total de 339 individuos contestaron al cuestionario. De esta muestra, 208 (61.5\%) fueron mujeres y 130 (38.5\%) hombres. Una persona no contestó a esta cuestión. La media de edad fue aproximadamente de 42 años $(M=41.54$; $S D=12.28)$. De acuerdo a la edad de los participantes se establecieron nueves grupos, de entre 20 a 24 años $(n=36 ; 10.7 \%), 25$ a 29 años $(n=37 ; 11 \%), 30$ a 34 años $(n=31 ; 9.3 \%), 35$ a 39 años $(n=33 ; 9.9 \%), 40$ a 44 años $(n=43 ; 12.8 \%)$, 45 a 49 años $(n=56 ; 16.7 \%), 50$ a 54 años $(n=39$; $11.6 \%), 55$ a 59 años $(n=39 ; 11.6)$, y 60 a 64 años $(n=21 ; 6.3 \%)$.

A nivel de formación académica, se observó que el $62.54 \%$ de los participantes $(n=212)$ poseía formación superior (grado, licenciatura, máster o doctorado) y el $37.56 \%(n=127)$ tenía estudios de secundaria o bachillerato.

Por sectores profesionales, la muestra estuvo compuesta por: administrativos y sector servicios $(n=109 ; 32 \%)$, gestores y directivos $(n=82 ; 23 \%)$, sanitarios $(n=66 ; 20 \%)$, comerciales $(n=46 ; 14 \%)$, y profesionales de los campos jurídico, industrial y económico (abogados, ingenieros, economistas) $(n=36 ; 11 \%)$.

\section{Instrumento de evaluación}

El cuestionario de Capital Psicológico-12 es la versión abreviada del Cuestionario de Capital Psicológico-24 (PCQ-24) de Luthans et al. (2007). Esta versión se compone de 12 ítems con seis opciones de respuesta $(1$ - Totalmente en desacuerdo; 2 - En desacuerdo; 3 - En parte de acuerdo; 4 - Algo de acuerdo; 5 - De acuerdo; 6 - Totalmente de acuerdo). El instrumento posee 
cuatro dimensiones: autoeficacia, esperanza, resiliencia y optimismo, cada una con un número específico de ítems. La esperanza se evalúa con cuatro ítems, (e.g., Actualmente estoy alcanzado los objetivos profesionales que me había marcado), autoeficacia (e.g., Me siento seguro/a al contribuir a los debates sobre la estrategia de la empresa) y resiliencia (e.g., Puedo trabajar "solo/a", sin ayuda, en mi trabajo si es necesario) con tres ítems, y el optimismo con dos ítems (e.g., En lo relativo a mi trabajo, siempre miro el lado bueno de las cosas).

\section{Procedimiento}

En primer lugar se contactó con los autores del instrumento a través del sitio web (www.mindgarden.com) para solicitar autorización de uso del PCQ-12 (Luthans et al., 2007). Una vez que se concedió el permiso comenzó el proceso de traducción siguiendo los trabajos de van Widenfelt, Treffers, Beurs, Siebelink y Koudijs (2005).

De esta forma, se recurrió a una metodología de traducción-retroversión. Inicialmente se procedió a una traducción del PCQ-12 de su lengua original (inglés) a la lengua española. A continuación se realizó una nueva traducción inversa de este instrumento. El siguiente paso implicó una comparación entre la versión original del PCQ-12 y la versión traducida. La traducción fue elaborada por un juez independiente bilingüe y bicultural. A su vez, la traducción inversa fue efectuada recurriendo a otro juez, igualmente bilingüe y bicultural. La comparación entre las dos versiones obtenidas se produjo en presencia de los jueces y sirvió para resolver divergencias a nivel del vocabulario utilizado. Por último, si se realiza un pre test a 50 personas con el objetivo de comprobar y mejorar la traducción.

La aplicación del instrumento se realizó en el lugar de trabajo y con la presencia de unos de los autores del trabajo. Se trata de un diseño metodológico de tipo trasversal, ya que la recogida de datos se realizó en un único momento. Con respecto a la amplitud de la muestra y aunque no existe consenso acerca del número de participantes necesarios para llevar a cabo un análisis factorial confirmatorio (AFC), seguimos los presupuestos de Lloret-Segura, FerreresTraver, Hernández-Baeza y Tomás-Marco (2014). Así, se buscó una muestra superior a 200 participantes para probar las calidades psicométricas del PCQ-12, en este caso se recogió una muestra compuesta por 339 trabajadores.

\section{Análisis de Datos}

Este instrumento tiene una estructura factorial predeterminada, por lo que el análisis estadístico se inició con un AFC realizado con el software estadístico Analysis of Moment Structures (AMOS) versión 20. Antes de este análisis se comprobó la distribución normal multivariada. Curran, West y Finch (1996) plantean para el método de estimación de máxima verosimilitud (MV), que valores de asimetría y curtosis de dos y siete, respectivamente, indican la existencia de una distribución normal multivariada. Nuestros resultados cumplieron con estos valores, por lo que el CFA podía llevarse a cabo (Tabla 1).

Tabla 1. Análisis Estadístico Uni y Multivariado

\begin{tabular}{lcccc}
\multicolumn{5}{c}{$(\mathrm{N}=339)$} \\
\hline Item & $M$ & $D T$ & $A$ & $C$ \\
\hline 1 & 4.11 & 1.49 & -.64 & -.59 \\
2 & 3.84 & 1.48 & -.40 & -.83 \\
3 & 4.14 & 1.41 & -.63 & -.39 \\
4 & 4.77 & 1.01 & -1.23 & 2.25 \\
5 & 4.16 & 1.26 & -.73 & .01 \\
6 & 4.37 & 1.16 & -.74 & .16 \\
7 & 3.97 & 1.31 & -.57 & -.35 \\
8 & 4.53 & 1.49 & -.99 & .04 \\
9 & 5.28 & .89 & -1.67 & 3.81 \\
10 & 4.89 & 1.14 & -1.14 & 1 \\
11 & 4.89 & 1.14 & -1.19 & 1.43 \\
12 & 4.59 & 1.34 & -1.08 & .53
\end{tabular}

Nota. $M=$ Media $D T=$ Desviación Típica; $A=$ Índice de Asimetría; $\mathrm{C}=$ Índice de Curtosis.

Tres modelos de ajuste fueron sometidos a análisis, un modelo de primer orden (Modelo 1), un modelo de segundo orden (Modelo 2) compuesto por cuatro factores (autoeficacia, esperanza, resiliencia, y optimismo) con un factor de segundo orden llamado Capital Psicológico, y un tercer modelo compuesto por cuatro factores correlacionados (Modelo 3).

Además de la prueba de ajuste de Chicuadrado $\left(\chi^{2}\right)$, el índice de ajuste comparativo (CFI), el error cuadrático medio de aproximación (RMSEA), y el residuo cuadrático medio (SRMR) fueron evaluados. Un CFI entre .90 y .95 es considerado bueno, y valores superiores a .95 muy buenos (Byrne, 2010; Marôco, 2010). Un ajuste aceptable para el RMSEA se logra con valores entre .10 y .05 , los valores inferiores a .05 son 

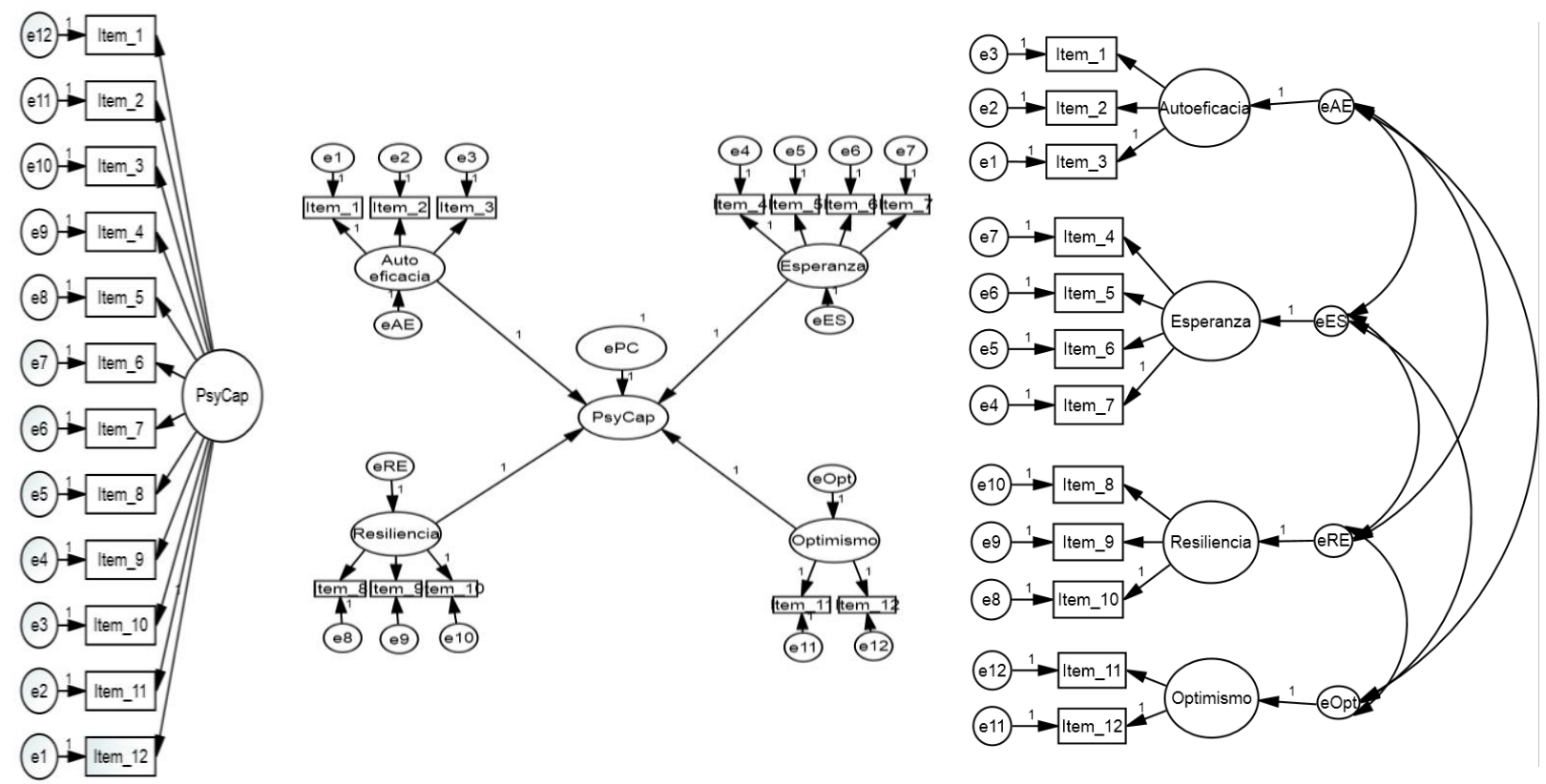

Figura 1. Modelos de ajuste probados. De izquierda a derecha, modelo de primer orden (modelo 1), modelo de segundo orden (modelo 2) y modelo con cuatro factores correlacionados (modelo 3 ).

Tabla 2. Indices de Ajuste para los Modelos factoriales Contrastados (N=339)

\begin{tabular}{lcccccc}
\hline Modelo & $\chi^{2 \mathrm{a}}$ & $\mathrm{df}^{\mathrm{b}}$ & RMSEA $^{\mathrm{c}}$ & $90 \% \mathrm{RMSEA}^{\mathrm{d}}$ & SRMR $^{\mathrm{e}}$ & $\mathrm{CFI}^{\mathrm{f}}$ \\
\hline Modelo 1 & $441.666^{* *}$ & 54 & .146 & $.133-.158$ & .087 & .749 \\
Modelo 2 & $497.961^{* *}$ & 55 & .154 & $.142-.167$ & .263 & .713 \\
Modelo 3 & $165.782^{* *}$ & 48 & .085 & $.071-.010$ & .057 & .924
\end{tabular}

Nota. ${ }^{* *} p<.01$; ${ }^{\text {a }}$ Prueba de bondad de ajuste de Chi Cuadrado; ${ }^{\mathrm{b}}$ Grados de libertad; ${ }^{\mathrm{c}}$ Raíz Cuadrada Media de Error de Aproximación ${ }^{\mathrm{d}}$ Raíz Cuadrada Media de Error de Aproximación con un intervalo de confianza del 90\%; ${ }^{\mathrm{e}}$ Raíz cuadrada Media Residual Estandarizada; ${ }^{\mathrm{f}}$ Indice de Ajuste Comparativo.

considerados buenos (Byrne, 2010; Marôco, 2010). Para este índice también se calculó un intervalo de confianza del $90 \%$ (90\% RMSEA). Un SRMR igual o inferior a .08 puntos representa un buen ajuste (Hu \& Bentler, 1999).

Los modelos fueron comparados a través del $\Delta \mathrm{CFI}$, las diferencias superiores a .01 significan que los modelos son estadísticamente diferentes (Cheung \& Rensvold, 2002). Para analizar si el ajuste de los modelos se podría mejorar, se evaluaron los índices de modificación. Asimismo, las cargas factoriales de los ítems se clasificaron según la propuesta de Comrey y Lee (1992), excelente (>.71); Muy bueno (>.63); Bueno (>.55); Aceptable (>.45); y pobre (>.32).

En términos de fiabilidad, se consideraron dos coeficientes, Alpha de Cronbach y Fiabilidad Compuesta, y se consideró la clasificación de Sharma (1996), que indica que valores superiores a .90 son excelentes; entre .80 y .90 son muy buenos; entre .70 y .80 son adecuados; y por debajo de .50 son pobres.

\section{Resultados}

Los resultados del análisis inicial a las estructuras factoriales propuestas demostraron que los modelos 1 y 2 presentaron un ajuste pobre, y que el modelo 3 tenía un ajuste aceptable (Tabla 2).

Todas las cargas de los factores fueron estadísticamente significativas y tuvieron el signo esperado. La menor carga se registró en el ítem 9 (Modelo 1) $(\lambda=.34)$ (Tabla 3).

A través del análisis de los índices de modificación se identificaron las covarianzas entre los errores de los ítems para mejorar el ajuste de los modelos. Se marcaron cuatro pares de ítems, ítems 1 y 2 , ítems 1 y 3 , ítems 4 y 7 , e 
Tabla 3. Factores de Carga de los Ítems del PCQ-12 en los Modelos Propuestos (N=339)

\begin{tabular}{|c|c|c|c|c|c|c|}
\hline \multirow{2}{*}{ Items } & \multicolumn{2}{|c|}{ Modelo 1} & \multicolumn{2}{|c|}{ Modelo 2} & \multicolumn{2}{|c|}{ Modelo 3} \\
\hline & $\lambda^{\mathrm{a}}$ & $\delta^{\mathrm{b}}$ & $\lambda$ & $\delta$ & $\lambda$ & $\delta$ \\
\hline Autoeficacia & - & - & $\begin{array}{c}.65 \\
(.87 ; .87)^{\mathrm{c}}\end{array}$ & - & $(.87 ; .87)$ & - \\
\hline Item 1 & .75 & .56 & .90 & .81 & .88 & .78 \\
\hline Item 2 & .75 & .56 & .84 & .71 & .85 & .73 \\
\hline Item 3 & .70 & .50 & .75 & .57 & .77 & .59 \\
\hline Esperanza & - & - & $\begin{array}{c}.30 \\
(.80 ; .81)\end{array}$ & - & $(.80 ; .81)$ & - \\
\hline Item 4 & .61 & .37 & .62 & .38 & .65 & .42 \\
\hline Item 5 & .72 & .52 & .83 & .69 & .81 & .65 \\
\hline Item 6 & .62 & .38 & .72 & .52 & .70 & .50 \\
\hline Item 7 & .67 & .44 & .68 & .47 & .70 & .49 \\
\hline Resiliencia & - & & $\begin{array}{c}.28 \\
(.50 ; .56)\end{array}$ & - & $(.50 ; .54)$ & - \\
\hline Item 8 & .45 & .20 & .40 & .16 & .48 & .23 \\
\hline Item 9 & .34 & .12 & .70 & .49 & .55 & .30 \\
\hline Item 10 & .37 & .14 & .52 & .27 & .57 & .33 \\
\hline Optimismo & - & & $\begin{array}{c}.41 \\
(.64 ; .65)\end{array}$ & - & $(.64 ; .65)$ & - \\
\hline Item 11 & .50 & .25 & .75 & .56 & .73 & .53 \\
\hline Item 12 & .48 & .23 & .64 & .41 & .66 & .43 \\
\hline PsyCap & $(.86 ; .86)$ & - & & - & - & - \\
\hline
\end{tabular}

Tabla 4. Indices de Ajuste para los Modelos Factoriales Reestructurados (N=339)

\begin{tabular}{rccccrc}
\hline Modelo & $\chi^{2 \mathrm{a}}$ & $\mathrm{df}^{\mathrm{b}}$ & RMSEA $^{\mathrm{c}}$ & $90 \% \mathrm{RMSEA}^{\mathrm{d}}$ & SRMR $^{\mathrm{e}}$ & $\mathrm{CFI}^{\mathrm{f}}$ \\
\hline Modelo 1 & $232.563^{* *}$ & 50 & .104 & $.091-.118$ & .068 & .882 \\
Modelo 2 & $482.462^{* *}$ & 54 & .153 & $.141-.166$ & .263 & .723 \\
Modelo 3 & $129.516^{* *}$ & 46 & .073 & $.059-.088$ & .055 & .946
\end{tabular}

Nota. ${ }^{* *} p<.01 ;{ }^{a}$ Prueba de bondad de ajuste de Chi Cuadrado; ${ }^{\mathrm{b}}$ Grados de libertad; ${ }^{\mathrm{c}}$ Raíz Cuadrada Media de Error de Aproximación ${ }^{\mathrm{d}}$ Raíz Cuadrada Media de Error de Aproximación con un intervalo de confianza del $90 \%$; ${ }^{\mathrm{e}} \mathrm{Raíz}$ cuadrada

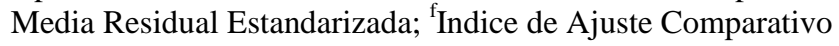

ítems 11 y 12. Posteriormente, los tres modelos se volvieron a analizar con la inclusión de estas covarianzas (Tabla 4).

Como puede observarse, sólo el Modelo 3 (cuatro factores correlacionados) presentó el mejor ajuste para todos los índices considerados, en comparación con los modelos 1 y 2. Del análisis de la Tabla 5 se desprende que el ítem 9 del modelo $1(\lambda=.38)$ es el que tiene la menor carga factorial.

El único modelo que cumplió con los valores de corte para todos los índices fue el modelo 3 (Tabla 5). Dado estos resultados, el $\Delta$ CFI no se calculó, ya que sólo un modelo, el modelo 3, alcanzó los valores requeridos para ser considerado como un modelo con buen ajuste. A través del análisis del modelo 3 se observa que existe una carga factorial (ítem 5: $\lambda=.46$ ) más baja. Sin embargo, la misma es considerada, de acuerdo con el enfoque de Comrey y Lee (1992), como aceptable. Así, se optó por mantener la totalidad de los ítems del PCQ-12, y también en consonancia con otros estudios del mismo instrumento (Rus et al., 2012; Viseu et al., 2012) que encontraron cargas factoriales similares y mantuvieron todos los ítems.

En cuanto a la fiabilidad, los valores obtenidos oscilaron entre .64 y .87 , es decir, de aceptable a muy buena (Sharma, 1996). La excepción se observó en la dimensión de resiliencia, 
Tabla 5. Factores de carga de los Ítems del PCQ-12 en los Modelos Reestructurados (N=339)

\begin{tabular}{|c|c|c|c|c|c|c|}
\hline \multirow{2}{*}{ Items } & \multicolumn{2}{|c|}{ Modelo 1} & \multicolumn{2}{|c|}{ Modelo 2} & \multicolumn{2}{|c|}{ Modelo 3} \\
\hline & $\lambda^{\mathrm{a}}$ & $\delta^{\mathrm{b}}$ & $\lambda$ & $\delta$ & $\lambda$ & $\delta$ \\
\hline Autoeficacia & - & - & $.57(.87 ; .83)^{\mathrm{c}}$ & - & $(.87 ; .84)$ & - \\
\hline Item 1 & .62 & .39 & .77 & .59 & .78 & .61 \\
\hline Item 2 & .63 & .39 & .78 & .61 & .82 & .67 \\
\hline Item 3 & .60 & .36 & .82 & .67 & .78 & .60 \\
\hline Esperanza & - & - & $\begin{array}{c}.34 \\
(.80 ; .82)\end{array}$ & - & $(.80 ; .82)$ & - \\
\hline Item 4 & .69 & .47 & .67 & .45 & .70 & .50 \\
\hline Item 5 & .78 & .60 & .79 & .63 & .78 & .61 \\
\hline Item 6 & .69 & .47 & .72 & .51 & .69 & .48 \\
\hline Item 7 & .73 & .53 & .73 & .54 & .75 & .56 \\
\hline Resiliencia & - & & $\begin{array}{c}.29 \\
(.50, .56)\end{array}$ & - & $(.50 ; .55)$ & - \\
\hline Item 8 & .40 & .16 & .40 & .16 & .46 & .21 \\
\hline Item 9 & .38 & .15 & .70 & .49 & .55 & .30 \\
\hline Item 10 & .41 & .16 & .42 & .27 & .59 & .35 \\
\hline Optimismo & - & & $\begin{array}{c}.49 \\
(.64 ; .80)\end{array}$ & & $(.64 ; .79)$ & - \\
\hline Item 11 & .52 & .27 & .88 & .77 & .87 & .76 \\
\hline Item 12 & .49 & .24 & .74 & .55 & .75 & .56 \\
\hline PsyCap & $(.86 ; .86)$ & - & & - & - & - \\
\hline
\end{tabular}

modelos 2 y 3, coincidente con lo encontrado en la versión portuguesa del PCQ-12 (Viseu et al., 2012). Al igual que en otros estudios se calculó el índice de fiabilidad global para este instrumento. Los valores obtenidos variaron entre .86 (coeficiente Alpha de Cronbach) y .92 (Fiablidad Compuesta) sugiriendo una consistencia interna de muy buena y excelente, respectivamente (Sharma, 1996).

\section{Discusión}

Este trabajo ha llevado a cabo un estudio psicométrico del cuestionario de capital psicológico positivo PCQ-12 en la población española mediante la técnica del análisis factorial y examinado su validez de constructo. De los tres modelos evaluados, el que presenta mejores resultados de ajuste es el modelo de correlación entre los cuatro factores, lo que sugiere que cada una de las dimensiones del capital psicológico: autoeficacia, esperanza, resiliencia y optimismo, tienen una entidad propia y están interrelacionadas entre sí.

Estos resultados no concuerdan con lo encontrado por otros autores que también han estudiado la validez de constructo del cuestionario PCQ-12 (León-Pérez et al., 2017; Rus et al., 2012; Wernsing, 2014), aunque sí coinciden con los encontrados por Viseu et al. (2012).

Los índices de consistencia interna se consideran entre muy buenos y adecuados para los factores de autoeficacia, esperanza y optimismo, siendo poco adecuados para el caso de resiliencia, en la línea con lo encontrado por Viseu et al. (2012). Los bajos valores de fiabilidad obtenidos para la dimensión resiliencia pueden deberse al reducido número de elementos que esta dimensión presenta. Es de destacar que en el trabajo de revisión psicométrica de Dawkins et al. (2013) se señala que lo estudios analizados muestran una menor consistencia interna para las dimensiones de optimismo (oscilando entre .63-.69) y resiliencia (oscilando entre .63-.66), y que tienden a ser consistentemente más bajas que las 
encontradas para la autoeficacia y esperanza, como ocurre en nuestro caso.

Valores distintos de consistencia interna (e.g., Léon-Pérez et al., 2017) se registraron utilizando el PCQ-12, sin embargo Rus et al. (2012) sugieren que la fiabilidad de un instrumento puede variar de estudio a otro, debiendo valorar los resultados obtenidos en el AFC. Este hecho es también evidenciado por Urbina (2004). Debido a las variaciones en los valores de consistencia interna, varios autores (e.g., Avey, Avolio, \& Luthans, 2011, Norman, Avey, Nimnicht, \& Pigeon, 2010, Rus et al., 2012, Woolley, Caza y Levy, 2011) han optado por aportar el valor de fiabilidad total para el PCQ-12. Al realizar este procedimiento verificamos que la fiabilidad de nuestro instrumento fue superior a .70. De acuerdo con la clasificación de Sharma (1996), los valores obtenidos variaron entre muy bueno (coeficiente Alpha de Cronbach) y excelente (Fiabilidad Compuesta).

El modelo que presentó un mejor ajuste (cuatro factores correlacionados) presentó un ítem con una carga factorial más baja. Sin embargo, recurriendo a la clasificación de Comrey y Lee (1992), la misma era clasificada como aceptable, lo que justifica la pertinencia de su mantenimiento en el PCQ-12. Otros autores (e.g., Rus et al., 2012, Viseu et al., 2012) optaron, igualmente, por la permanencia de ítems con cargas factoriales más reducidas, lo que permite la preservación de la identidad de este instrumento y facilita la realización de estudios comparativos.

Los estudios de validación del PCQ-12 no son tan concluyentes como los del PCQ-24, y algunos autores apuntan a la existencia de diferencias culturales en la medida del capital psicológico evaluado con este instrumento. Así en el estudio de Wernsing (2014), con una muestra superior a 56.000 empleados y 12 muestras de países diferentes, encontró problemas en la medida de invarianza en términos de convergencias y dependencias entre los factores de primer orden. Aunque cada muestra se ajustó al modelo factorial de segundo orden, la misma autora señaló diferentes patrones de ajuste. Las muestras de Alemania, Suecia, Reino Unido y Estado Unidos obtuvieron mejores ajustes al modelo original, mientras que Italia, Turquía, Polonia y China, este último caso en concordancia con estudios previos
(Luthans et al., 2008), obtuvieron un menor nivel y requirieron ajuste adicionales. Una de las limitaciones señaladas por la autora es que a pesar de tener una muestra muy amplia correspondía por entero a una misma organización, y que la cultura organizacional podría influir en los resultados, esta misma limitación podría señalarse en el estudio de León-Pérez et al. (2017). Nuestra muestra supera esa limitación al ser muy heterogénea y estar constituida por trabajadores de diversos sectores y ámbitos (tanto público como privado).

El capital psicológico se revela como un importante elemento del comportamiento organizacional positivo con un significativo desarrollo empírico en cuanto a su validez de constructo y validez externa, pero que necesita más investigación para comprender su medida y como contribuye cada uno de sus componentes.

En ese sentido las implicaciones y contribuciones de este trabajo son las siguientes. Este trabajo evidencia la existencia de una estructura factorial distinta a la propuesta por los autores del constructo que contribuye a la investigación y desafíos planteados en la investigación actual sobre el Capital Psicológico Positivo en relación a la posible influencia de variables culturales, su conceptualización y estructura factorial. Por una parte, nuestro modelo de cuatro factores correlacionados coincide con lo encontrado por otros autores con muestra de diferentes países (Viseu et al., 2012) lo que evidencia la posible existencia e influencia de diferencias culturales y sectoriales en la estructura factorial del Capital Psicológico Positivo. Por otro lado, diferentes estudios han señalado las dificultades para lograr un modelo de segundo orden con los ítems y factores planteados. En algunos casos lograr ese ajuste implicó eliminar ítems por errores en la medida de covarianza (Rego et al., 2010). En otros casos, como en la investigación de Wersing (2014), con una muestra de 12 países, se encontraron diferencias en términos de ajuste y fiabilidad en los resultados de cada país, hasta el punto de proponer una nueva versión de 9 ítems, a la que llamó cross-cultural PCQ-9, en la que eliminaba un ítem de la dimensión esperanza y la dimensión optimismo (dos ítems). Esta es precisamente una de las dimensiones que en 11 de los 16 estudios 
consultados en el completo análisis de las propiedades psicométricas del constructo de Capital Psicológico, llevado a cabo por Dawkins et al (2013), obtiene índices de consistencia interna más bajos, como también ocurre en nuestro estudio. Estos autores señalan la necesidad de reconceptualizar este factor.

Este estudio aporta también evidencia en relación a estudios previos sobre la consistencia interna de la dimensión resiliencia y en concreto al ítem 9. Es esta dimensión la que menor consistencia interna muestra en los diferentes estudios consultados (Dawkins et al., 2013). Así mismo es destacable que en nuestro estudio sea el ítem 9 (dimensión resiliencia) el que presente una carga factorial más baja, en la línea de la encontrado por los autores que han llevado a cabo otros estudios factoriales del PCQ en población española (Azanza et al., 2014; León et al., 2017) lo que apuntaría a la necesidad de abordar la evaluación y redefinición de ese ítem.

En conclusión, este trabajo aporta evidencia en cuanto a la existencia de una estructura factorial distinta del Capital Psicológico Positivo, apoyada por resultados de estudios previos que apuntarían la influencia de diferencias culturales y del entorno y sector organizacional. Apoya la evidencia sobre la consistencia interna de cada uno de los factores en el constructo de Capital Psicológico Positivo, y plantea la necesidad de revisión de determinados ítems para población española.

En cuanto a sus limitaciones, se trata de una muestra no representativa y de carácter trasversal de la población española, por lo que futuros estudios deberían mejorar y apuntar en esta dirección. Se debería trabajar en investigaciones de tipo longitudinal y diversos sectores, pues el Capital Psicológico como estado psicológico de desarrollo positivo debería verse influenciado por la experiencia. Además aportaría claridad a otro de los desafíos actuales como es su papel como variable mediadora y moderadora del rendimiento laboral, sin olvidar su influencia en la percepción de estrés y bienestar psicológico. Otra de las prioridades de investigaciones futuras debería orientarse hacia los estudios interculturales, de modo que se analice el ajuste de los modelos y las diferencias entre el promedio de los ítems, así como analizar la influencia de la cultura organizacional y otros factores de nivel organizacional.

\section{Referencias}

Alessandri, G., Borgogni, L., Consiglio, C., \& Mitidieri, G. (2015). Psychometric properties of the Italian version of the psychological capital questionnaire. International Journal of Selection and Assessment, 23, 149-159. doi:10.1111/ijsa.12103

Avey, J. (2014). The left side of psychological capital: New evidence on the antecedents of PsyCap. Journal of Leadership \& Organizational Studies, 21, 141-149. doi:10.1177/1548051813515516

Avey, J., Avolio, B., \& Luthans, F. (2011). Experimentally analyzing the impact of leader positivity on follower positivity and performance. The Leadership Quarterly, 22, 282-294. doi:10.1016/j.leaqua.2011.02.004

Avey, J. B., Luthans, F., \& Youssef, C. M. (2010). The additive value of positive psychological capital in predicting work attitudes and behaviors. Journal of Management, 36, 430452. doi: 10.1177/0149206308329961

Avey, J. B., Reichard, R. J., Luthans, F., \& Mhatre, K. H. (2011). Meta-analysis of the impact of positive psychological capital on employee attitudes, behaviors, and performance. Human Resource Development Quarterly, 22, 127-152. doi: 10.1002/hrdq. 20070

Avey, J. B., Wernsing, T. S., \& Luthans, F. (2008). Can positive employees help positive organizational change? Impact of psychological capital and emotions on relevant attitudes and behaviors. Journal of Applied Behavioral Science, 44, 48-70. doi:10.1177/0021886307311470

Azanza, G., Domínguez, Á. J., Moriano, J. A., \& Molero, F. J. (2014). Capital psicológico positivo: Validación del cuestionario PCQ en España [Positive psychological capital [Validation of the Spanish version of the PCQ questionnaire]. Anales de Psicología, 30, 294301. doi:10.6018/analesps.30.1.153631

Bakker, A. B., \& Demerouti, E. (2013). La teoría de las demandas y los recursos laborales [Job demands-resources model]. Revista de 
Psicología del Trabajo y de las Organizaciones, 29, 107-115. doi:10.5093/tr2013a16

Bakker, A. B., \& Rodríguez-Muñoz, A. (2012). Introducción a la psicología de la salud ocupacional positiva [Introduction to the positive psychology of occupational health]. Psicothema, 24, 62-65.

Badran, M. A., \& Youssef-Morgan, C. M. (2015). Psychological capital and job satisfaction in Egypt. Journal of Managerial Psychology, 30, 354-370. doi:10.1108/JMP-06-2013-0176

Bandura, A. (1997). Self-efficacy: The exercise of control. New York, NY: Freeman.

Baron, R. A., Franklin, R. J., \& Hmieleski, K. M. (2013). Why entrepreneurs often experience low, not high, levels of stress: The joint effects of selection and psychological capital. Journal of Management, 42, 742-768. doi:10.1177/0149206313495411

Barrera-Guzmán, M. L., \& Flores-Galaz, M. M. (2015). Construcción de una Escala de Salud Mental Positiva para Adultos en Poblacion Mexicana. Revista Iberoamericana de Diagnóstico y Evaluación - e Avaliação Psicológica, 1, 22-33.

Block, J., \& Kremen, A.M. (1996). IQ and egoresiliency: Conceptual and empirical connections and separateness. Journal of Personality and Social Psychology, 70, 349361. doi:10.1037/0022-3514.70.2.349

Byrne, B. (2010). Structural equation modeling with AMOS: Basic concepts, application, and programing ( $2^{\text {nd }}$ Ed.). New York, NY: Routledge.

Cabanas, E., \& Sanchez-González, J.C. (2016). Inverting the pyramid of needs: Positive psychology's new order for labor success. Psicothema, 28, 107-113.

Caza, A., Bagozzi, R. P., Woolley, L., Levy, L., \& Caza, B. B. (2010). Psychological capital and authentic leadership: Measurement, gender, and cultural extension. AsiaPacific Journal of Business Administration, 2, 53-70. doi:10.1108/17574321011028972

Cheung, G., \& Rensvold, R. (2002). Evaluating goodness-of-fit indexes for testing measurement invariance. Structural Equation Modeling, 9, 233-255. doi:10.1207/S1532800SEM0902_5
Comrey, A., \& Lee, H. (1992). A first course in factor analysis $\left(2^{\text {nd }}\right.$ ed.). New York, NY: Erlbaum.

Curran, P., West, S., \& Finch, J. (1996). The robustness of test statistics to nonnormality and specification error in confirmatory factor analysis. Psychological Methods, 1, 16-29. doi: 10.1037/1082-989X.1.1.16

Dawkins, S., Martin, A., Scott, J., \& Sanderson, K. (2013). Building of the positives: A psychometric review and critical analysis of the construct of psychological capital. Journal of Occupational and Organizational Psychology, 86, 348-370. doi:10.1111/joop.12007

Hu, L., \& Bentler, P. (1999). Cutoff criteria for fit indexes in covariance structure analysis: Conventional criteria versus new alternatives. Structural Equation Modeling, 6, 1-55. doi:10.1080/10705519909540118

León-Pérez, J. M., Antino, M., \& León-Rubio, J. M. (2017). Adaptation of the short version of the Psychological Capital Questionnaire (PCQ-12) into Spanish. Revista de Psicología Social, 1-18.

Lloret-Segura, S., Ferreres-Traver, A., HernándezBaeza, A., \& Tomás-Marco, I. (2014). El análisis factorial exploratorio de los ítems: Una guía práctica, revisada y actualizada. Anales de Psicología, 30, 1151-1169. doi:10.6018/analesps.30.3.199361

Luthans, F. (2002a). The need for and meaning of positive organizational behavior. Journal of Organizational Behavior, 23. 695-706. doi:10.1080/02134748.2016.1248024

Luthans, F. (2002b). Positive organizational behavior: Developing and managing psychological strengths. Academy of Management Executive, 16, 57-72. doi:10.5465/AME.2002.6640181

Luthans, F., Avey, J. B., Clapp-Smith, R., \& Li, W. (2008). More evidence on the value of Chinese workers' psychological capital: A potentially unlimited competitive resource? International Journal of Human Resource Management, 19, 818-827. doi:10.1080/09585190801991194

Luthans, F., Youssef-Morgan, C. M., \& Avolio, B. J. (2015). Psychological capital and 
beyond. Oxford, UK: Oxford University Press.

Luthans, F., Avolio, B., Avey, J. B., \& Norman, S. M. (2007). Psychological capital: Measurement and relationship with performance and job satisfaction. Personnel Psychology, 60, 541-572. doi:10.1111/j.1744-6570.2007.00083.x

Luthans, F., \& Ibrayeva, E. S. (2006). Entrepreneurial self-efficacy in Central Asian transition economies: Quantitative and qualitative analyses. Journal of International Business Studies, 37, 92-110. doi: 10.1057/palgrave.jibs. 8400173

Luthans, K. W., \& Jensen, S. M. (2005). The linkage between psychological capital and commitment to organizational mission: A study of nurses. Journal of Nursing Administration, 35(6), 304-310

Luthans, F., Youssef, C., \& Avolio, B. (2007). Psychological capital: Developing the human competitive edge. New York, NY: Oxford University Press.

Marôco, J. (2010). Análise de equaçõesestruturais: Fundamentosteóricos, software e aplicações. Pêro Pinheiro, Portugal: ReportNumber.

Martínez, P., \& Cassaretto, M. (2012). Propiedades psicométricas de la Escala de Esperanza de Herth en español. Revista Iberoamericana de Diagnóstico y Evaluación Psicológica, 33(1), 127-145.

Maslow, A. (1954). Motivation and personality. New York, NY: Harper.

Myers, D. (2000). The funds, friends and faith of happy people. American Psychologist, 55, 5667. doi:10.1037/0003-066X.55.1.56

Newman, A., Ucbasaran, D., Zhu, F., \& Hirst, G. (2014). Psychological capital: A review and synthesis. Journal of Organizational Behavior, 35(S1), S120-S138. doi:10.1002/job.1916

Norman, S., Avey, J., Nimnicht, J., \& Pigeon, N. (2010). The interactive effect of psychological capital and organizational identity on employee organizational citizenship and deviance behaviors. Journal of Leadership \& Organizational Studies, 17, 380-391.

doi:10.1177/1548051809353764
Parker, S. (1998). Enhancing role-breadth selfefficacy: The roles of job enrichment and other organizational interventions. Journal of Applied Psychology, 83, 835-852. doi: 10.1037//0021-9010.83.6.835

Peterson, S. J., \& Luthans, F. (2003). The positive impact and development of hopeful leaders. Leadership and Organization Development Journal, 24, 26-31. doi:10.1108/01437730310457302

Peterson, C., \& Steen, T. (2002). Optimistic explanatory style. In C. R. Snyder \& S. Lopez (Eds.), Handbook of positive psychology (pp. 244-256). Oxford, UK: Oxford University Press.

Rego, A., Marques, C., Leal, S., Sousa, F., \& Pina e Cunha, M. (2010). Psychological capital and performance of Portuguese civil servants: exploring neutralizers in the context of an appraisal system. The International Journal of Human Resource Management, 21, 15311552. doi:10.1080/09585192.2010.488459

Rogers, C. R. (1961). On becoming a person. Boston, MA: Houghton Mifflin.

Rus, C., Baban, A., Jesus, S. N., \& Andrei, D. (2012). An analysis of the psychometric properties of the Psychological Capital Questionnaire-12. Journal of Educational Sciences \& Psychology, 1, 110-122.

Sánchez-Teruel, D., \& Robles-Bello, M. A. (2015). Escala de Resiliencia 14 ítems (RS14): propiedades psicométricas de la versión en Español. Revista Iberoamericana de Diagnóstico y Evaluación - e Avaliação Psicológica, 40, 103-113.

Scheier, M. F., \& Carver, C. S. (1985). Optimism, coping, and health: Assessment and implications of generalized outcome expectancies. Health Psychology, 4, 219-247. doi:10.1037/0278-6133.4.3.219

Schulman, P. (1999). Applying learned optimism to increase sales productivity. Journal of Personal Selling and Sales Management, 19, 31-37. doi:10.1080/08853134.1999.10754157

Seligman, M. E. P., \& Csikszentmihalyi, M. (2000). Positive psychology: An introduction. American Psychologist, 55, 5-14. doi:10.1037/0003-066X.55.1.5 
Sharma, S. (1996). Applied multivariate techniques. New York, NY: John Wiley and Sons Inc.

Snyder, C. R., Irving, L., \& Anderson, J. (1991). Hope and health. In C. R. Snyder \& D. R. Forsyth (Eds.), Handbook of social and clinical psychology (pp. 285-305). Elmsford, NY: Pergamon.

Snyder, C. R., Sympson, S., Ybasco, F., Borders, T., Babyak, M., \& Higgins, R. (1996). Development and validation of the state hope scale. Journal of Personality and Social Psychology, 70, 321-335. doi:10.1037/00223514.70.2.321

Stajkovic, A., \& Luthans, F. (1998). Social cognitive theory and self-efficacy: Going beyond traditional motivational and behavioral aspects. Organizational Dynamics, 26(4), 62-74. doi:10.1016/S0090-2616(98)90006-7

Sweetman, D., Luthans, F., Avey, J. B., \& Luthans, B. C. (2011). Relationship between positive psychological capital and creative performance. Canadian Journal of Administrative Sciences/Revue Canadienne des Sciences de l'Administration, 28(1), 4-13.

Torrente, P., Salanova, M., Llorens, S., \& Schaufeli, W.B. (2012). Teams make it work: How team work engagement mediates between social resources and performance in teams. Psicothema, 24, 106-112.

Urbina, S. (2004). Essentials of psychological testing. Hoboken, NJ: John Wiley \& Sons.

Van Widenfelt, B., Treffers, P., Beurs, E., Siebelink, B. \& Koudijs, E. (2005). Translation and cross-cultural adaptation of assessment instruments used in psychological research with children and families. Clinical Child and Family Psychology Review, 8, 135147. doi:10.1007/s10567-005-4752-1

Viseu, J., Jesus, S. N., Rus, C., Nunes, H., Lobo, P., \& Cara-Linda, I. (2012). Psychological capital and its' assessement by PCQ-12. Estudos Contemporâneos da Subjectividade, 2, 4-16.

Wagnild, G. M., \& Young, H. M. (1993). Development and psychometric evaluation of the resilience scale. Journal of Nursing Management, 1, 165-178.
Waite, P., \& Richardson, G. (2004). Determining the efficacy of resiliency training in the work site. Journal of Allied Health, 33, 178-183.

Wernsing, T. (2014). Psychological Capital: A test of measurement invariance across 12 national cultures. Journal of Leadership \& Organizational Studies, 21, 179-190. doi:10.1177/154805181351924

Woolley, L., Caza, A., \& Levy, L. (2011). Authentic leadership and follower development: Psychological capital, positive work climate, and gender. Journal of Leadership \& Organizational Studies, 18, 438-448. doi: 10.1177/1548051810382013

Youssef-Morgan, C. (2014). Advancing OB research: An illustration using psychological capital. Journal of Leadership \& Organizational Studies, 21, 130-140. doi:10.1177/1548051813515512

Youssef, C. M., \& Luthans, F. (2011). Positive psychological capital in the workplace: Where we are and where we need to go. In K. M. Sheldon, T. B. Kashdan \& M. F. Steger (Eds.), Designing positive psychology: Taking stock and moving forward (pp. 351-364). New York, NY:Oxford University Press.

Youssef, C. M., \& Luthans, F. (2012). Psychological capital: Meaning, findings and future directions.In K. S. Cameron \& G. M. Spreitzer (Eds.), The Oxford handbook of positive organizational scholarship (pp. 1727). Oxford, UK: Oxford University Press.

Youssef-Morgan, C., \& Luthans, F. (2015). Psychological capital and well-being. Stress and Health, 31, 180-188. doi:10.1002/smi.2623 